\title{
ANALYSIS AND NUMERICAL SIMULATION OF MAGNETIC FORCES BETWEEN RIGID POLYGONAL BODIES. PART I: ANALYSIS
}

\author{
NIKOLA POPOVIĆ, DIRK PRAETORIUS, AND ANJA SCHLÖMERKEMPER
}

\begin{abstract}
The mathematical and physical analysis of magnetoelastic phenomena is a topic of ongoing research. Different formulae have been proposed to describe the magnetic forces in macroscopic systems. We discuss several of these formulae in the context of rigid magnetized bodies. In case the bodies are in contact, we consider formulae both in the framework of macroscopic electrodynamics and via a multiscale approach, i.e., in a discrete setting of magnetic dipole moments. We give mathematically rigorous proofs for domains of polygonal shape (as well as for more general geometries) in two and three space dimensions. In an accompanying second article, we investigate the formulae in a number of numerical experiments, where we focus on the dependence of the magnetic force on the distance between the bodies and on the case when the two bodies are in contact. The aim of the analysis as well as of the numerical simulation is to contribute to the ongoing debate about which formula describes the magnetic force between macroscopic bodies best and to stimulate corresponding real-life experiments.
\end{abstract}

\section{INTRODUCTION}

The analysis and modeling of magnetoelastic phenomena is a topic of ongoing research in both mathematics and physics. One aspect of the analysis concerns formulae for the magnetic force acting between rigid bodies, on which we focus throughout the present article. The force which is exerted by a magnetic field on a single magnetic dipole is well understood; similarly, there is no controversy regarding the total acting magnetic force between macroscopic rigid magnetized bodies that are a positive distance apart, i.e., not in contact.

By contrast, formulae for the magnetic force acting inside macroscopic magnetized systems have been under discussion for quite a time, cf. e.g. [Bob00, Bro66, DPG96, EM90]. For the magnetic force acting between two portions of one rigid magnetized body, different formulae were derived within the context of macroscopic electrodynamics. Similarly, for the case of two magnetized bodies that are in contact, one can find various force formulae in the literature. This has resulted in some controversy. We refer to [Bob00] for a clarifying exposition: Several of the formulae occurring in the literature are physically equivalent if one considers appropriate sets of physical fields, see also [Bro66, Dör68, DPG96].

In the context of rigid magnetized bodies considered here, however, the force formulae differ due to intrinsic differences in the modeling: One approach is based on macroscopic electrodynamics, whereas the other sets out from a discrete setting of magnetic dipole moments of which the continuum limit is calculated. This latter approach leads to an additional contribution to the force, which is due to interactions of dipoles close to the interface of the two bodies in contact. Since we assume that the bodies are rigid and that no external fields are present, we believe that no

Date: May 3, 2007.

Key words and phrases. Magnetostatics, magnetic force formulae, potential theory, partial differential equations, lattice-to-continuum limit.

2006 PACS. 41.20.Gz, 45.20.da, 02.30.Em. 
additional force terms of magnetostatic origin have to be taken into account; see the discussion in [PPS] for details.

The question which of the formulae under consideration is the most appropriate one in that it describes nature best has not been resolved yet. The aim of this article as well as of its companion paper [PPS] is to contribute to the ongoing debate by studying several of these formulae, and the relation between them, both analytically and numerically. In particular, we hope that our study will stimulate clarifying experimental work.

To the best of our knowledge, it is not possible to measure the magnetic force within magnetized bodies, i.e., the force exerted by one portion of a magnetic body on its ("nested") complement. We therefore study a different setup, which seems to be realizable in the context of real-life experiments. The results of these experiments should also give some insight into the question which formula describes the force within magnetized bodies most accurately. More specifically, the setup considered in this article is the following: Let $A$ and $B$ be two magnetized rigid bodies, with magnetization $\mathbf{m}$, in two or three space dimensions. We assume that $A$ and $B$ are either a positive distance apart or that they are in contact. However, they do not have to be nested, i.e., we do not require $\bar{A} \subset B$, where $\bar{A}$ denotes the closure of $A$ under the usual Euclidean norm on $\mathbb{R}^{d}(d=2,3)$.

If the distance between $A$ and $B$ is greater than zero, we are concerned primarily with the classical force formula $\mathbf{F}(A, B)=\int_{A}(\mathbf{m} \cdot \nabla) \mathbf{H}_{B} d x$, see e.g. [Bob00, Bro66]. Here, $\mathbf{H}_{B}$ is the magnetic field generated by the magnetization in $B$ which is given as the solution of the corresponding stationary Maxwell equations, cf. Section 2. (Similarly, the fields generated by $\mathbf{m}_{A}$ and $\mathbf{m}_{A \cup B}$ will be denoted by $\mathbf{H}_{A}$ and $\mathbf{H}_{A \cup B}$, respectively.) In [PPS], we investigate $\mathbf{F}(A, B)$ in a series of numerical experiments in which the force is computed in dependence on the distance between $A$ and $B$.

At the end of Section 2, we briefly discuss another force formula for this setting, namely $\widetilde{\mathbf{F}}(A, B)=\int_{A}(\mathbf{m} \cdot \nabla) \mathbf{H}_{A \cup B} d x$, which is for instance considered in [LL84]. This formula differs from $\mathbf{F}(A, B)$ by a term which depends only on the specific assumptions on $A$ and $\mathbf{m}_{A}$ and which is hence independent of $B$ and $\mathbf{m}_{B}$ as well as of the distance between $A$ and $B$.

If the distance between $A$ and $B$ is zero, i.e., if $A$ and $B$ are in contact, we take two different approaches. First, we consider and rigorously prove a force formula which was extensively discussed by Brown [Bro66], cf. Theorem 3.1. We denote the corresponding formula by $\mathbf{F}^{\mathrm{Br}}$. The proof provided here is a generalization of a result in [Sch02, Theorem 2.1] from $\mathcal{C}^{2}$ domains to Lipschitz domains with piecewise $\mathcal{C}^{1,1}$ boundaries in two and three dimensions; the domains are now assumed to be in contact, but are not necessarily nested. (Here, $\mathcal{C}^{2}$ and $\mathcal{C}^{1,1}$ are appropriately defined spaces of twice continuously differentiable functions and of differentiable functions with Lipschitz continuous derivatives, respectively.) Moreover, we establish the following relation between $\mathbf{F}$ and $\mathbf{F}^{\mathrm{Br}}$ : Let $B_{\varepsilon}$ be a shifted copy of $B$ such that the distance between $A$ and $B_{\varepsilon}$ is $\varepsilon$. Then, we show that $\mathbf{F}^{\mathrm{Br}}(A, B)$ is the limit of $\mathbf{F}\left(A, B_{\varepsilon}\right)$ as the distance between $A$ and $B_{\varepsilon}$ tends to zero.

Secondly, we consider a force formula, $\mathbf{F}^{\mathrm{lim}}$, which is derived from a discrete setting of magnetic dipole moments. The resulting expression contains an additional surface force term, called $\mathbf{F}^{\text {short }}$, which reflects the structure of the underlying lattice. The derivation of the corresponding continuum limit is based on methods adapted from [Sch05], where it is assumed that $A$ and $B$ are threedimensional and nested. In Theorems 3.3 and 3.4, we generalize the results of [Sch05] to the case of two magnetic polygonal Lipschitz domains $A$ and $B$ in $\mathbb{R}^{2}$ or $\mathbb{R}^{3}$ which are in contact but not necessarily nested. (For an extension of these results to more general domains, cf. Remark 3.7 below.)

From a mathematical point of view, the generalization of both $\mathbf{F}^{\mathrm{Br}}$ and $\mathbf{F}^{\mathrm{lim}}$ in the present setting requires stronger results on the regularity of the corresponding solutions of Maxwell's equations 
and a stronger statement on lattice approximations of surface integrals than is required for the analysis of the "nested" setting, cf. Proposition 3.5; these are provided in [Schb, Scha].

Finally, we refer to [PPS] for the numerical simulation of the above formulae as well as for a physical interpretation. In particular, in Section 4 of [PPS] we collect the conclusions of our study, and we comment on open topics for future research as well as on related analytical questions.

\section{Separated Magnetic Bodies}

In this section, we review a (well-known) classical formula for the magnetic force acting between two separated magnetized bodies. To be precise, we make the following assumptions throughout: Let $A, B \subset \mathbb{R}^{d}(d=2,3)$ be given, where the requirement that $A$ and $B$ are separated implies $\bar{A} \cap \bar{B}=\emptyset$, and let $\varepsilon:=\operatorname{dist}(A, B)>0$ denote their (Euclidean) distance. We assume that $A$ and $B$ are bounded Lipschitz domains in $\mathbb{R}^{d}$ with polygonal boundaries and finitely many corners or edges. As it turns out, these assumptions are sufficient for the numerical experiments performed in [PPS]; moreover, they allow us to easily apply regularity results from [Scha]. However, we note that they could in principle be relaxed, cf. also Remark 3.7.

Let $\mathbf{m}_{A}$ and $\mathbf{m}_{B}$ denote the magnetization fields on $A$ and $B$. We assume that $\mathbf{m}_{A}: A \rightarrow \mathbb{R}^{d}$ and $\mathbf{m}_{B}: B \rightarrow \mathbb{R}^{d}$ are Lipschitz continuous vector fields that are supported on $\bar{A}$ and $\bar{B}$, respectively, i.e., there holds $\mathbf{m}_{A} \in W^{1, \infty}(A)$ and $\mathbf{m}_{B} \in W^{1, \infty}(B)$. For technical reasons, we extend $\mathbf{m}_{A}$ and $\mathbf{m}_{B}$ by zero to the entire space $\mathbb{R}^{d}$. The magnetic force $\mathbf{F}(A, B)$ which is exerted by $B$ on $A$ is given by

$$
\mathbf{F}(A, B)=\int_{A}\left(\mathbf{m}_{A}(x) \cdot \nabla\right) \mathbf{H}_{B}(x) d x=\int_{A} \sum_{j=1}^{d}\left(\mathbf{m}_{A}\right)_{j}(x) \partial_{j} \mathbf{H}_{B}(x) d x
$$

(see e.g. [Bob00, Bro66]), where $\left(\mathbf{m}_{A}\right)_{j}, 1 \leq j \leq d$ denotes the $j$-th component of $\mathbf{m}_{A}$ and $\mathbf{H}_{B}$ is the magnetic field obtained from the magnetostatic Maxwell equations

$$
\operatorname{div} \mathbf{B}_{B}=0, \quad \operatorname{curl} \mathbf{H}_{B}=0, \quad \mathbf{B}_{B}-\mathbf{H}_{B}=\gamma \mathbf{m}_{B},
$$

i.e., we assume that there are no conduction currents present. Here, $\mathbf{B}_{B}$ denotes the magnetic induction, and $\gamma$ is a constant which depends only on the choice of physical units: In Gaussian units, there holds for instance $\gamma=4 \pi$, whereas $\gamma=1$ corresponds to the Lorentz-Heaviside unit system, cf. [Bro66, p. 6]. According to the Stokes Theorem, the magnetic field reads $\mathbf{H}_{B}=-\nabla u_{B}$, where the magnetic potential $u_{B}: \mathbb{R}^{d} \rightarrow \mathbb{R}$ is the solution of the corresponding Poisson equation stated in weak form as

$$
\operatorname{div}\left(-\nabla u_{B}+\gamma \mathbf{m}_{B}\right)=0 \quad \text { in } \mathcal{D}^{\prime}\left(\mathbb{R}^{d}\right):=\left(\mathcal{C}_{c}^{\infty}\left(\mathbb{R}^{d}\right)\right)^{\prime} ;
$$

here, $\mathcal{D}\left(\mathbb{R}^{d}\right)=\mathcal{C}_{c}^{\infty}\left(\mathbb{R}^{d}\right)$ denotes the space of compactly supported functions on $\mathbb{R}^{d}$ and $\mathcal{D}^{\prime}\left(\mathbb{R}^{d}\right)$ is its dual space. Note that, as a consequence, we have the transition conditions $\left[\nabla u_{B} \cdot \mathbf{n}_{B}\right]=-\gamma \mathbf{m}_{B} \cdot \mathbf{n}_{B}$ as well as $\left[u_{B}\right]=0$ on $\partial B$, with $\mathbf{m}_{B}$ the inner trace of $\mathbf{m}_{B}$ with respect to $B, \mathbf{n}_{B}$ the outer normal vector on $\partial B$, and [.] the difference between the outer and the inner trace. In particular, for $x \notin \partial B$ the magnetic field $\mathbf{H}_{B}$ has the integral representation

$$
\mathbf{H}_{B}(x)=-\gamma \int_{B}\left(-\nabla \cdot \mathbf{m}_{B}\right)(y) \nabla N(x-y) d y-\gamma \int_{\partial B}\left(\mathbf{m}_{B} \cdot \mathbf{n}_{B}\right)(y) \nabla N(x-y) d s_{y},
$$

where $s_{y}$ denotes the surface measure, see e.g. [ES98, p. 73]. Here, $\mathbf{m}_{B}$ is understood in the sense of traces. (This convention will be applied throughout the article; if nothing else is specified, the trace is always taken from inside the boundary.) Moreover, the Newtonian kernel $N(\cdot)$ and its gradient 
$\nabla N$ are given by

$$
N(x):=\left\{\begin{array}{ll}
-\frac{1}{2 \pi} \log |x| & \text { for } d=2, \\
\frac{1}{4 \pi} \frac{1}{|x|} & \text { for } d=3
\end{array} \quad \text { and } \quad \nabla N(x)= \begin{cases}-\frac{1}{2 \pi} \frac{x}{|x|^{2}} & \text { for } d=2, \\
-\frac{1}{4 \pi} \frac{x}{|x|^{3}} & \text { for } d=3,\end{cases}\right.
$$

respectively. Note that under the above assumptions, there holds $\mathbf{H}_{B} \in W^{1,1}(A)$, see [Scha].

Since the distance $\varepsilon$ between $A$ and $B$ is positive, we can substitute (2.2) into (2.1) and integrate by parts to obtain

$$
\begin{aligned}
\mathbf{F}(A, B)=\int_{A} & \left(-\nabla \cdot \mathbf{m}_{A}\right)(x)\left(-\gamma \int_{B}\left(-\nabla \cdot \mathbf{m}_{B}\right) \nabla N(x-y) d y\right. \\
& \left.-\gamma \int_{\partial B}\left(\mathbf{m}_{B} \cdot \mathbf{n}_{B}\right)(y) \nabla N(x-y) d s_{y}\right) d x \\
+ & \int_{\partial A}\left(\mathbf{m}_{A} \cdot \mathbf{n}_{A}\right)(x)\left(-\gamma \int_{B}\left(-\nabla \cdot \mathbf{m}_{B}\right) \nabla N(x-y) d y\right. \\
& \left.-\gamma \int_{\partial B}\left(\mathbf{m}_{B} \cdot \mathbf{n}_{B}\right)(y) \nabla N(x-y) d s_{y}\right) d s_{x} .
\end{aligned}
$$

In the numerical experiments in [PPS], we will restrict ourselves to magnetization fields $\mathbf{m}_{A}$ and $\mathbf{m}_{B}$ that are constant on $A$ and $B$, respectively. Then, all terms in (2.3) except for the last one vanish. We quote the corresponding formula for this particular case for later reference here:

$$
\mathbf{F}_{\text {const }}(A, B)=-\gamma \int_{\partial A}\left(\mathbf{m}_{A} \cdot \mathbf{n}_{A}\right)(x) \int_{\partial B}\left(\mathbf{m}_{B} \cdot \mathbf{n}_{B}\right)(y) \nabla N(x-y) d s_{y} d s_{x}
$$

Remark 2.1. Though we are usually interested in small distances, we want to comment briefly on the behavior of $|\mathbf{F}(A, B)|$ for $\varepsilon$ large. Since $|x-y|>\operatorname{dist}(A, B)$ for all $x \in A$ and $y \in B$, we have $|\nabla N(x-y)| \leq c|x-y|^{1-d} \leq c \operatorname{dist}(A, B)^{1-d}$ for some generic constant $c$. Hence, (2.3) can be estimated by $c \operatorname{dist}(A, B)^{1-d}$, since $\nabla \cdot \mathbf{m}_{A}, \nabla \cdot \mathbf{m}_{B}, \mathbf{m}_{A} \cdot \mathbf{n}_{A}$ and $\mathbf{m}_{B} \cdot \mathbf{n}_{B}$ are bounded by assumption. This estimate is well-known for $d=3$ : For large distances, the magnetic force between two bodies decreases like $\operatorname{dist}(A, B)^{-2}$.

This bound on the force, however, certainly is not accurate as the distance between the bodies tends to zero and would correspond to an infinitely strong force which is not observed in nature. The force between two bodies in contact is strong, but not infinitely so, see also [PPS].

As already mentioned in the introduction, we want to consider briefly another force formula for positive distances which is discussed in the literature, $\widetilde{\mathbf{F}}(A, B)=\int_{A}\left(\mathbf{m}_{A} \cdot \nabla\right) \mathbf{H}_{A \cup B} d x$. A calculation which is similar to the one that gives (3.3) below yields

$$
\begin{aligned}
\widetilde{\mathbf{F}}(A, B) & =\int_{A}\left(\mathbf{m}_{A} \cdot \nabla\right) \mathbf{H}_{B} d x-\frac{\gamma}{2} \int_{\partial A}\left(\mathbf{m}_{A} \cdot \mathbf{n}_{A}\right)^{2} \mathbf{n}_{A} d s_{x} \\
& =\mathbf{F}(A, B)-\frac{\gamma}{2} \int_{\partial A}\left(\mathbf{m}_{A} \cdot \mathbf{n}_{A}\right)^{2} \mathbf{n}_{A} d s_{x},
\end{aligned}
$$

see also [LL84, Equation (35.4)]. Here, the term $\frac{\gamma}{2} \int_{\partial A}\left(\mathbf{m}_{A} \cdot \mathbf{n}_{A}\right)^{2} \mathbf{n}_{A} d s_{x}$ does not depend on the distance between $A$ and $B$, but only on the specification of $A$ and $\mathbf{m}_{A}$. In the numerical experiments in [PPS], we always assume a uniform magnetization $\mathbf{m}_{A}$. Due to our assumptions on the geometries of the magnetic bodies under consideration, we have $\frac{\gamma}{2} \int_{\partial A}\left(\mathbf{m}_{A} \cdot \mathbf{n}_{A}\right)^{2} \mathbf{n}_{A} d s_{x}=0$ and, hence, $\mathbf{F}(A, B)=\widetilde{\mathbf{F}}(A, B)$. 


\section{Magnetic Bodies in Contact}

In this section, we discuss two force formulae for the case when the two magnetized bodies are in contact. Let $A$ and $B$ be two domains in $\mathbb{R}^{d}$, with positive surface measure $\partial A \cap \partial B$, and denote the corresponding magnetizations by $\mathbf{m}_{A}$ and $\mathbf{m}_{B}$, respectively. For ease of presentation and in view of the upcoming application in [PPS], we make the following assumptions throughout this section. All these assumptions are essentially of a technical nature and are inherited from the results of [Schb, Scha] used in the proofs below.

Assumption $\mathcal{A}$. Let $d=2$ or $d=3$ be fixed.

(1) $A$ and $B$ are bounded Lipschitz domains in $\mathbb{R}^{d}$ with polygonal boundaries and finitely many corners or edges such that $A \cap B=\emptyset$. Moreover, $A$ and $B$ are in contact, i.e., the surface measure of $\partial A \cap \partial B \subset \partial A$ is positive;

(2) the corresponding magnetizations $\mathbf{m}_{A}: A \rightarrow \mathbb{R}^{d}$ and $\mathbf{m}_{B}: B \rightarrow \mathbb{R}^{d}$ are Lipschitz continuous and are supported on $\bar{A}$ and $\bar{B}$, respectively, i.e., there holds $\mathbf{m}_{A} \in W^{1, \infty}(A)$ and $\mathbf{m}_{B} \in$ $W^{1, \infty}(B)$. Moreover, $\mathbf{m}_{A}$ and $\mathbf{m}_{B}$ are extended by zero to the entire space $\mathbb{R}^{d}$.

In Remark 3.7, we will briefly comment on how the above assumptions on $A$ and $B$ can be relaxed. We consider two formulae for the force acting between $A$ and $B$ in the following, namely $\mathbf{F}^{\mathrm{Br}}$ and $\mathbf{F}^{\mathrm{lim}}$, which are stated in Equations (3.3) and (3.26) below.

3.1. Brown's Force Formula $\mathbf{F}^{\mathrm{Br}}$. The formula which we refer to as Brown's force formula was analyzed extensively by Brown [Bro66] in the form

$$
\mathbf{F}^{\mathrm{Br}}(A, B):=\int_{A}\left(\mathbf{m}_{A}(x) \cdot \nabla\right) \mathbf{H}_{A \cup B}(x) d x+\frac{\gamma}{2} \int_{\partial A}\left(\mathbf{m}_{A}(x) \cdot \mathbf{n}_{A}(x)\right)^{2} \mathbf{n}_{A}(x) d s_{x} .
$$

Here, $\mathbf{H}_{A \cup B}=-\nabla u_{A \cup B}$ denotes the magnetic field generated by the magnetization $\mathbf{m}_{A \cup B}=$ $\mathbf{m}_{A}+\mathbf{m}_{B}$. The potential $u_{A \cup B}: \mathbb{R}^{d} \rightarrow \mathbb{R}$ is a solution of

$$
\operatorname{div}\left(-\nabla u_{A \cup B}+\gamma \mathbf{m}_{A \cup B}\right)=0 \quad \text { in } \mathcal{D}^{\prime}\left(\mathbb{R}^{d}\right),
$$

which implies that the transition conditions

$$
\left[\nabla u_{A \cup B} \cdot \mathbf{n}_{A}\right]=\gamma\left(\mathbf{m}_{B}-\mathbf{m}_{A}\right) \cdot \mathbf{n}_{A} \quad \text { and } \quad\left[u_{A \cup B}\right]=0 \quad \text { on } \partial A
$$

(with $\mathbf{m}_{B}=0$ on $\partial A \backslash(\partial A \cap \partial B)$ ) as well as

$$
\left[\nabla u_{A \cup B} \cdot \mathbf{n}_{B}\right]=\gamma\left(\mathbf{m}_{A}-\mathbf{m}_{B}\right) \cdot \mathbf{n}_{B} \quad \text { and } \quad\left[u_{A \cup B}\right]=0 \quad \text { on } \partial B
$$

(with $\mathbf{m}_{A}=0$ on $\partial B \backslash(\partial A \cap \partial B)$ ) must hold.

For three-dimensional nested bodies with $\mathcal{C}^{2}$ boundaries, it is shown in [Sch02] that Brown's force formula equals $\int_{A}\left(\mathbf{m}_{A}(x) \cdot \nabla\right) \mathbf{H}_{B}(x) d x$ and, hence, that $\mathbf{F}^{\mathrm{Br}}$ is equivalent to the classical formula (2.1) if that formula is applied formally to the case when $A$ and $B$ are in contact. In the following theorem, we prove that this assertion is also valid in our geometrical setting, both in two and in three dimensions.

Theorem 3.1. Under Assumption $\mathcal{A}$, there holds

$$
\mathbf{F}^{\mathrm{Br}}(A, B)=\int_{A}\left(\mathbf{m}_{A}(x) \cdot \nabla\right) \mathbf{H}_{B}(x) d x .
$$

Proof. By the linearity of Maxwell's equations we have $\mathbf{H}_{B}=\mathbf{H}_{A \cup B}-\mathbf{H}_{A}$. Thus,

$$
\int_{A}\left(\mathbf{m}_{A} \cdot \nabla\right) \mathbf{H}_{B} d x=\int_{A}\left(\mathbf{m}_{A} \cdot \nabla\right) \mathbf{H}_{A \cup B} d x-\int_{A}\left(\mathbf{m}_{A} \cdot \nabla\right) \mathbf{H}_{A} d x
$$


where the existence of the integrals follows from $\nabla \mathbf{H}_{A}, \nabla \mathbf{H}_{A \cup B} \in L^{1}(A)$ [Scha, Theorem 16]. Hence, we only need to show that

$$
\mathbf{F}_{0}:=\int_{A}\left(\mathbf{m}_{A}(x) \cdot \nabla\right) \mathbf{H}_{A}(x) d x=-\frac{\gamma}{2} \int_{\partial A}\left(\mathbf{m}_{A} \cdot \mathbf{n}_{A}\right)^{2}(x) \mathbf{n}_{A}(x) d s_{x} .
$$

An integration by parts yields

$$
\mathbf{F}_{0}=\int_{A}\left(-\nabla \cdot \mathbf{m}_{A}\right)(x) \mathbf{H}_{A}(x) d x+\int_{\partial A}\left(\mathbf{m}_{A} \cdot \mathbf{n}_{A}\right)(x) \mathbf{H}_{A}^{-}(x) d s_{x} .
$$

Here, $\mathbf{H}_{A}$ is the gradient of the solution of the corresponding Poisson equation, which can be represented in analogy to $(2.2)$ :

$$
\mathbf{H}_{A}(x)=-\gamma \int_{A}\left(-\nabla \cdot \mathbf{m}_{A}\right)(y) \nabla N(x-y) d y-\gamma \int_{\partial A}\left(\mathbf{m}_{A} \cdot \mathbf{n}_{A}\right)(y) \nabla N(x-y) d s_{y}
$$

for almost every $x \in A$ and in $L^{1}(A)$ [Scha, Theorem 13]. For $x \in \partial A$, a study of the respective gradients of the Newton potential and the single-layer potential yields that the inner trace of $\mathbf{H}_{A}$ with respect to $A$ is given by

$$
\begin{aligned}
\mathbf{H}_{A}^{-}(x)= & -\gamma \int_{A}\left(-\nabla \cdot \mathbf{m}_{A}\right)(y) \nabla N(x-y) d y-\gamma \oint_{\partial A}\left(\mathbf{m}_{A} \cdot \mathbf{n}_{A}\right)(y) \nabla N(x-y) d s_{y} \\
& -\frac{\gamma}{2}\left(\mathbf{m}_{A} \cdot \mathbf{n}_{A}\right)(x) \mathbf{n}_{A}(x)
\end{aligned}
$$

for almost every $x \in \partial A$ and in $L^{1}(\partial A)$, cf. [Scha, Theorem 13]. Here, $\oint_{\partial A}(\cdot) d s_{y}$ denotes the Cauchy principal value integral, i.e., $\oint_{\partial A} f(x-y) d s_{y}$ is shorthand for $\lim _{\delta \rightarrow 0} \int_{\partial A \backslash B_{\delta}(x)} f(x-y) d s_{y}$, and $B_{\delta}(x)$ is the $d$-dimensional ball of radius $\delta>0$ about $x$.

By Assumption $\mathcal{A}$, we have $\nabla \cdot \mathbf{m}_{A} \in L^{\infty}(A)$ and $\mathbf{m}_{A} \cdot \mathbf{n}_{A} \in L^{\infty}(\partial A)$. Since $\mathbf{H}_{A} \in L^{1}(A)$ and $\mathbf{H}_{A}^{-} \in L^{1}(\partial A)$, the integrals in (3.5) exist, and we can substitute the integral representations of $\mathbf{H}_{A}$ and $\mathbf{H}_{A}^{-}$into (3.5), which gives

$$
\begin{aligned}
\mathbf{F}_{0}= & \int_{A}\left(-\nabla \cdot \mathbf{m}_{A}\right)(x)\left(-\gamma \int_{A}\left(-\nabla \cdot \mathbf{m}_{A}\right)(y) \nabla N(x-y) d y\right. \\
& \left.-\gamma \int_{\partial A}\left(\mathbf{m}_{A} \cdot \mathbf{n}_{A}\right)(y) \nabla N(x-y) d s_{y}\right) d x \\
+ & \int_{\partial A}\left(\mathbf{m}_{A} \cdot \mathbf{n}_{A}\right)(x)\left(-\gamma \int_{A}\left(-\nabla \cdot \mathbf{m}_{A}\right)(y) \nabla N(x-y) d y\right. \\
& \left.-\gamma \oint_{\partial A}\left(\mathbf{m}_{A} \cdot \mathbf{n}_{A}\right)(y) \nabla N(x-y) d s_{y}-\frac{\gamma}{2}\left(\mathbf{m}_{A} \cdot \mathbf{n}_{A}\right)(x) \mathbf{n}_{A}(x)\right) d s_{x} .
\end{aligned}
$$

Next, we apply Fubini's Theorem to the first term and change variables via $x \leftrightarrow y$. By the symmetry of the kernel, this yields $\gamma \int_{A}\left(-\nabla \cdot \mathbf{m}_{A}\right)(x) \int_{A}\left(-\nabla \cdot \mathbf{m}_{A}\right)(y) \nabla N(x-y) d y d x$, which is the negative of the above and hence is zero. An application of Fubini's Theorem to the second term and an exchange of the variables $x$ and $y$ shows that the second term is in fact the negative of the third. Therefore, these two terms cancel. By the definition of the Cauchy integral, the fourth term reads

$$
\int_{\partial A}\left(\mathbf{m}_{A} \cdot \mathbf{n}_{A}\right)(x) \lim _{\delta \rightarrow 0} \int_{\partial A \backslash B_{\delta}(x)}\left(\mathbf{m}_{A} \cdot \mathbf{n}_{A}\right)(y) \nabla N(x-y) d s_{y} d s_{x} .
$$

If $A$ were smooth, the underlying convergence would be uniform. However, in our case we still have convergence in $L^{1}(\partial A)$ by [Scha, Lemma 11]. Since $\mathbf{m}_{A} \cdot \mathbf{n}_{A} \in L^{\infty}(\partial A)$, the fourth term thus 
equals

$$
\lim _{\delta \rightarrow 0} \int_{\partial A}\left(\mathbf{m}_{A} \cdot \mathbf{n}_{A}\right)(x) \int_{\partial A \backslash B_{\delta}(x)}\left(\mathbf{m}_{A} \cdot \mathbf{n}_{A}\right)(y) \nabla N(x-y) d s_{y} d s_{x} .
$$

Again, an application of Fubini's Theorem and an exchange of the variables $x$ and $y$ shows that this term equals its negative; therefore it is zero. Hence $\mathbf{F}_{0}=-\frac{\gamma}{2} \int_{\partial A}\left(\mathbf{m}_{A} \cdot \mathbf{n}_{A}\right)^{2} \mathbf{n}_{A} d s_{x}$, which concludes the proof.

As an aside, we mention that Equation (3.4) verifies what is to be expected on physical grounds: As pointed out by [DPG96], $\int_{A}\left(\mathbf{m}_{A}(x) \cdot \nabla\right) \mathbf{H}_{A}(x) d x+\frac{\gamma}{2} \int_{\partial A}\left(\mathbf{m}_{A} \cdot \mathbf{n}_{A}\right)^{2}(x) \mathbf{n}_{A}(x) d s_{x}$ can be interpreted as the self-force exerted by $A$ on itself, which then has to be zero by conservation of momentum.

In preparation for the numerical experiments presented in [PPS], we state Brown's formula separately under the additional assumption of constant magnetization fields.

Corollary 3.2. Provided that Assumption $\mathcal{A}$ holds and that $\mathbf{m}_{A}$ and $\mathbf{m}_{B}$ are constant on $A$ and $B$, respectively, it follows that

$$
\begin{aligned}
\mathbf{F}_{\text {const }}^{\mathrm{Br}}(A, B)= & -\gamma \int_{\partial A}\left(\mathbf{m}_{A} \cdot \mathbf{n}_{A}\right)(x) \oint_{\partial B}\left(\mathbf{m}_{B} \cdot \mathbf{n}_{B}\right)(y) \nabla N(x-y) d s_{y} d s_{x} \\
& +\frac{\gamma}{2} \int_{\partial A \cap \partial B}\left(\mathbf{m}_{A} \cdot \mathbf{n}_{A}\right)\left(\mathbf{m}_{B} \cdot \mathbf{n}_{A}\right) \mathbf{n}_{A} d s_{x} .
\end{aligned}
$$

Proof. By [Scha, Theorems 13 and 16], we have $\mathbf{H}_{B} \in W^{1,1}(A)$. Thus, Theorem 3.1 and an integration by parts yield

$$
\mathbf{F}_{\text {const }}^{\mathrm{Br}}(A, B)=\int_{\partial A}\left(\mathbf{m}_{A} \cdot \mathbf{n}_{A}\right)(x) \mathbf{H}_{B}^{-}(x) d s_{x} .
$$

Here, $\mathbf{H}_{B}^{-}$is the inner trace of $\mathbf{H}_{B}$ with respect to $A$, and is equal to the outer trace of $\mathbf{H}_{B}$ with respect to $B$. For $\mathbf{m}_{B}$ constant, we thus have in analogy to (3.6)

$$
\mathbf{H}_{B}^{-}(x)=-\gamma \oint_{\partial B}\left(\mathbf{m}_{B} \cdot \mathbf{n}_{B}\right)(y) \nabla N(x-y) d s_{y}+\frac{\gamma}{2}\left(\mathbf{m}_{B} \cdot \mathbf{n}_{B}\right)(x) \mathbf{n}_{B}(x)
$$

for almost every $x \in \partial A \cup \partial B$ and in $L^{1}(\partial A \cup \partial B)$, see again [Scha, Theorem 13]. Again, there holds $\mathbf{m}_{A} \cdot \mathbf{n}_{A} \in L^{\infty}(\partial A)$; therefore, the formula for $\mathbf{H}_{B}^{-}$can be used in (3.8). Then, the first term equals zero by a change of variables and Fubini's Theorem, cf. the proof of Theorem 3.1. Since $\left(\mathbf{m}_{B} \cdot \mathbf{n}_{B}\right) \mathbf{n}_{B}=\left(\mathbf{m}_{B} \cdot \mathbf{n}_{A}\right) \mathbf{n}_{A}$ on $\partial A \cap \partial B$, the assertion follows.

We now briefly discuss the relation between $\mathbf{F}^{\mathrm{Br}}$ from (3.1) and the classical force formula $\mathbf{F}$, see (2.1), where we recall that $\mathbf{F}$ is a priori defined for separated magnetic bodies only. We begin by indicating how Brown obtained his formula $\mathbf{F}^{\mathrm{Br}}$ in (3.1), cf. [Bro66, p. 53]. Brown was interested in deriving a formula for the force which is exerted by one part of a magnetized body on its complement. To that end, he considered two nested sets $A \subset B$. Since Brown did not want to naively apply the force formula (2.1) for separated bodies, he introduced an interface layer in order to separate the two bodies artificially. This allowed him to use the classical formula (2.1). Brown then obtained his formula (3.1) in the limit as the thickness of the interface layer tends to zero. As he pointed out, this approach neglects any short range contributions from dipole moments close to the interface. (Note that precisely these contributions are considered in Section 3.2 below.)

One can show that the limit of the force $\mathbf{F}$ is equal to Brown's force $\mathbf{F}^{\mathrm{Br}}$ as the distance between $A$ and $B$ tends to zero. We give a proof in the simplified setting considered in [PPS], i.e., we assume that $A, B, \mathbf{m}_{A}$, and $\mathbf{m}_{B}$ satisfy Assumption $\mathcal{A}$, but in addition also that $A$ and $B$ are rectangular (or cuboidal) and that for $\varepsilon=0$, they have a boundary segment in common. 
Let $B_{\varepsilon}$ be a shifted copy of $B$ such that $B_{\varepsilon}=\left\{x+\varepsilon e_{1} \mid x \in B\right\}$, where $e_{1}$ denotes the first unit vector $(1,0) \in \mathbb{R}^{2}$, respectively $(1,0,0) \in \mathbb{R}^{3}$. Then, $\varepsilon=\operatorname{dist}\left(A, B_{\varepsilon}\right)>0$, and there holds

$$
\begin{aligned}
\lim _{\varepsilon \rightarrow 0} \mathbf{F}\left(A, B_{\varepsilon}\right) & =\lim _{\varepsilon \rightarrow 0} \int_{A}\left(\mathbf{m}_{A}(x) \cdot \nabla\right) \mathbf{H}_{B_{\varepsilon}}(x) d x \\
& =\lim _{\varepsilon \rightarrow 0} \int_{A}\left(-\nabla \cdot \mathbf{m}_{A}\right)(x) \mathbf{H}_{B_{\varepsilon}}(x) d x+\lim _{\varepsilon \rightarrow 0} \int_{\partial A}\left(\mathbf{m}_{A} \cdot \mathbf{n}_{A}\right)(x) \mathbf{H}_{B_{\varepsilon}}(x) d s_{x} \\
& =\lim _{\varepsilon \rightarrow 0} \int_{A}\left(-\nabla \cdot \mathbf{m}_{A}\right)(x) \mathbf{H}_{B}\left(x-\varepsilon e_{1}\right) d x+\lim _{\varepsilon \rightarrow 0} \int_{\partial A}\left(\mathbf{m}_{A} \cdot \mathbf{n}_{A}\right)(x) \mathbf{H}_{B}\left(x-\varepsilon e_{1}\right) d s_{x} .
\end{aligned}
$$

Since the magnetic field $\mathbf{H}_{B}\left(x-\varepsilon e_{1}\right)$ converges to $\mathbf{H}_{B}(x)$ in $L^{1}(A)$ and to $\mathbf{H}_{B}^{-}(x)$ in $L^{1}(\partial A)$ as $\varepsilon \rightarrow 0$ and since, moreover, $\nabla \mathbf{H}_{B} \in L^{1}(A)$ by [Scha, Theorem 16], we conclude that

$$
\begin{aligned}
\lim _{\varepsilon \rightarrow 0} \mathbf{F}\left(A, B_{\varepsilon}\right) & =\int_{A}\left(-\nabla \cdot \mathbf{m}_{A}\right)(x) \mathbf{H}_{B}(x) d x+\int_{\partial A}\left(\mathbf{m}_{A} \cdot \mathbf{n}_{A}\right)(x) \mathbf{H}_{B}^{-}(x) d s_{x} \\
& =\int_{A}\left(\mathbf{m}_{A} \cdot \nabla\right) \mathbf{H}_{B}(x) d x \\
& =\mathbf{F}^{\mathrm{Br}}(A, B) .
\end{aligned}
$$

Remark 3.1. We take this opportunity to correct a wrong statement following Theorem 3.1 in [PPS05] which was unfortunately premature, resulting in misleading conclusions in [PPS05, Section 4]. The limiting behavior of $\mathbf{F}(A, B)$ as described above is correct, cf. also the numerical analysis in [PPS].

3.2. Force Formula $\mathbf{F}^{\text {lim }}$ in the Discrete-to-Continuum Limit. The formula $\mathbf{F}^{\text {lim }}$ is derived in the continuum limit of a discrete setting of magnetic dipoles. It was introduced and studied in [Sch02, MS02, Sch05] in the case when $A=\tau$ is a sub-body of a larger body $\Omega \subset \mathbb{R}^{3}$, with $B=\Omega \backslash \bar{\tau}$. Here, we state the corresponding results in the setting of Assumption $\mathcal{A}$. (In fact, it can be shown that these results also hold for the more general domains defined in Remark 3.7 below.) Note that the main ideas of the proofs given in [Sch02, MS02, Sch05] carry over. The required modifications are of a rather technical nature and are discussed in full detail in [Schb] and [Scha].

Let $\mathcal{L}$ denote an (underlying) Bravais lattice of the magnetic material under consideration, i.e., let $\mathcal{L}=\left\{x \in \mathbb{R}^{d} \mid x=\sum_{i=1}^{d} \mu_{i} e_{i}, \mu_{i} \in \mathbb{Z}\right\}$, where $\left\{e_{1}, \ldots, e_{d}\right\}$ is a basis of $\mathbb{R}^{d}$. For simplicity, we assume that the measure of the unit cell $\left\{x \in \mathbb{R}^{d} \mid x=\sum_{i=1}^{d} \lambda_{i} e_{i}, \lambda_{i} \in[0,1)\right\}$ equals one; we can for instance take $\mathcal{L}=\mathbb{Z}^{d}$. Moreover, let $\frac{1}{\ell} \mathcal{L}=\left\{z \in \mathbb{R}^{d} \mid \ell z \in \mathcal{L}\right\}$, for $\ell \in \mathbb{N}$, denote the corresponding scaled Bravais lattice. To each point of $\frac{1}{\ell} \mathcal{L}$ we assign a magnetic dipole moment $\mathbf{m}^{(\ell)}$, which is related to the magnetization by the scaling law

$$
\mathbf{m}^{(\ell)}(x):= \begin{cases}\mathbf{m}_{A}^{(\ell)}(x):=\frac{1}{\ell^{d}} \mathbf{m}_{A}(x) & \text { if } x \in A \cap \frac{1}{\ell} \mathcal{L}, \\ \mathbf{m}_{B}^{(\ell)}(x):=\frac{1}{\ell^{d}} \mathbf{m}_{B}(x) & \text { if } x \in B \cap \frac{1}{\ell} \mathcal{L} .\end{cases}
$$

By the superposition principle, the $k$-th component of the magnetic force exerted by dipoles in $B$ on those in $\bar{A}$ reads

$$
\mathbf{F}_{k}^{(\ell)}(A, B):=\gamma \sum_{x \in \bar{A} \cap \frac{1}{\ell} \mathcal{L}} \sum_{y \in B \cap \frac{1}{\ell} \mathcal{L}} \partial_{i} \partial_{j} \partial_{k} N(x-y)\left(\mathbf{m}_{A}^{(\ell)}\right)_{i}(x)\left(\mathbf{m}_{B}^{(\ell)}\right)_{j}(y)
$$

in the discrete setting, cf. e.g. [Sch05]. Here and in the following, we adopt the Einstein summation convention, i.e., indices which occur twice in a formula are summed over. (Hence, (3.10) contains sums over $i, j=1, \ldots, d$.) 
Let $\varphi^{(\delta)}$ be a smooth function such that

$$
\varphi^{(\delta)}(z)= \begin{cases}1 & \text { if }|z|<\frac{\delta}{2} \\ 0 & \text { if }|z|>\delta\end{cases}
$$

In order to be able to state the following theorems simultaneously in $\mathbb{R}^{2}$ and $\mathbb{R}^{3}$, we introduce the abbreviations

$$
\begin{aligned}
& P_{k}^{(\delta)}(x-y):= \begin{cases}\left(\varphi^{(\delta)} \partial_{k} N\right)(x-y) & \text { for } d=2, \\
\partial_{k}\left(\varphi^{(\delta)} N\right)(x-y) & \text { for } d=3,\end{cases} \\
& R_{k}^{(\delta)}(x-y):= \begin{cases}\left(\left(1-\varphi^{(\delta)}\right) \partial_{k} N\right)(x-y) & \text { for } d=2, \\
\partial_{k}\left(\left(1-\varphi^{(\delta)}\right) N\right)(x-y) & \text { for } d=3 .\end{cases}
\end{aligned}
$$

Note that clearly $P_{k}^{(\delta)}(x-y)+R_{k}^{(\delta)}(x-y)=\partial_{k} N(x-y)$.

Remark 3.2. The reason for the differing definitions of $P_{k}^{(\delta)}$ and $R_{k}^{(\delta)}$ in dependence on $d$ is the following: Recall that $N$ is defined differently for $d=2$ and $d=3$, respectively. However, by the above definitions, $\varphi^{(\delta)}$ is multiplied by a homogeneous function of degree -1 in both cases. This homogeneity is used in the proofs of (3.14) and (3.22) as well as in a scaling argument in (A.1) below.

With the help of $R_{k}^{(\delta)}$ and $P_{k}^{(\delta)}$, we can split $\mathbf{F}^{(\ell)}$ into two parts, which we call the long range part and the short range part, respectively:

$$
\begin{aligned}
\mathbf{F}_{k}^{(\ell)}(A, B)= & \gamma \sum_{x \in \bar{A} \cap \frac{1}{\ell} \mathcal{L}} \sum_{y \in B \cap \frac{1}{\ell} \mathcal{L}} \partial_{i} \partial_{j} R_{k}^{(\delta)}(x-y)\left(\mathbf{m}_{A}^{(\ell)}\right)_{i}(x)\left(\mathbf{m}_{B}^{(\ell)}\right)_{j}(y) \\
& +\gamma \sum_{x \in \bar{A} \cap \frac{1}{\ell} \mathcal{L}} \sum_{y \in B \cap \frac{1}{\ell} \mathcal{L}} \partial_{i} \partial_{j} P_{k}^{(\delta)}(x-y)\left(\mathbf{m}_{A}^{(\ell)}\right)_{i}(x)\left(\mathbf{m}_{B}^{(\ell)}\right)_{j}(y) \\
= & : \mathbf{F}_{k}^{\operatorname{long}(\ell, \delta)}(A, B)+\mathbf{F}_{k}^{\text {short }(\ell, \delta)}(A, B) .
\end{aligned}
$$

The continuum limit of $\mathbf{F}^{(\ell)}$ is obtained by the following limiting procedure,

$$
\begin{aligned}
\mathbf{F}^{\lim }(A, B) & :=\lim _{\ell \rightarrow \infty} \mathbf{F}^{(\ell)}(A, B)=\lim _{\delta \rightarrow 0} \lim _{\ell \rightarrow \infty} \mathbf{F}^{\operatorname{long}(\ell, \delta)}(A, B)+\lim _{\delta \rightarrow 0} \lim _{\ell \rightarrow \infty} \mathbf{F}^{\operatorname{short}(\ell, \delta)}(A, B) \\
& =: \mathbf{F}^{\operatorname{long}}(A, B)+\mathbf{F}^{\text {short }}(A, B),
\end{aligned}
$$

where the existence of the limits on the right hand side of (3.13) is proven in the subsequent Theorems 3.3 and 3.4. We first focus on the contribution coming from the long range part $\mathbf{F}^{\text {long: }}$

Theorem 3.3. Under Assumption $\mathcal{A}$, the limit $\mathbf{F}^{\operatorname{long}}(A, B):=\lim _{\delta \rightarrow 0} \lim _{\ell \rightarrow \infty} \mathbf{F}^{\operatorname{long}(\ell, \delta)}(A, B)$ exists, and there holds

$$
\mathbf{F}^{\text {long }}(A, B)=\int_{A}\left(\mathbf{m}_{A} \cdot \nabla\right) \mathbf{H}_{A \cup B} d x+\frac{\gamma}{2} \int_{\partial A}\left(\mathbf{m}_{A} \cdot \mathbf{n}_{A}\right)\left(\left(\mathbf{m}_{A}-\mathbf{m}_{B}\right) \cdot \mathbf{n}_{A}\right) \mathbf{n}_{A} d s_{x}
$$

where $\mathbf{m}_{B}$ denotes the outer trace on $\partial A$ with respect to $A$, i.e., $\mathbf{m}_{B}$ is equal to zero on $\partial A \backslash(\partial A \cap \partial B)$ and equals the inner trace of $\mathbf{m}_{B}$ on $\partial A \cap \partial B$ with respect to $B$.

Proof. The inner limit $\lim _{\ell \rightarrow \infty} \mathbf{F}^{\text {long }(\ell, \delta)}$ corresponds to replacing a Riemann sum by an integral,

$$
\begin{aligned}
\lim _{\ell \rightarrow \infty} \mathbf{F}_{k}^{\operatorname{long}(\ell, \delta)} & =\gamma \int_{A} \int_{B} \partial_{i} \partial_{j} R_{k}^{(\delta)}(x-y)\left(\mathbf{m}_{A}\right)_{i}(x)\left(\mathbf{m}_{B}\right)_{j}(y) d y d x \\
& =\gamma \int_{A}\left(\mathbf{m}_{A}\right)_{i}(x) \partial_{i}\left(\int_{A \cup B}\left(\mathbf{m}_{A \cup B}\right)_{j}(y) \partial_{j} R_{k}^{(\delta)}(x-y) d y\right) d x
\end{aligned}
$$


where the second equality follows by a symmetry argument, cf. the proof of Theorem 3.1. The outer limit of $\delta \rightarrow 0$ can be handled by a careful analysis of the relevant singular integrals: We set

$$
\left(\mathbf{H}_{A \cup B}^{(\delta)}\right)_{k}(x)=\gamma \int_{A \cup B}\left(\mathbf{m}_{A \cup B}\right)_{j}(y) \partial_{j} R_{k}^{(\delta)}(x-y) d y
$$

for all $x \in \mathbb{R}^{d}$; then, (3.15) gives

$$
\lim _{\ell \rightarrow \infty} \mathbf{F}^{\operatorname{long}(\ell, \delta)}=\int_{A}\left(-\nabla \cdot \mathbf{m}_{A}\right) \mathbf{H}_{A \cup B}^{(\delta)} d x+\int_{\partial A}\left(\mathbf{m}_{A} \cdot \mathbf{n}_{A}\right) \mathbf{H}_{A \cup B}^{(\delta)} d s_{x} .
$$

An integration by parts of (3.16) yields

$$
\begin{aligned}
\left(\mathbf{H}_{A \cup B}^{(\delta)}\right)_{k}(x)= & -\gamma \int_{A \cup B}\left(-\nabla \cdot \mathbf{m}_{A \cup B}\right)(y) R_{k}^{(\delta)}(x-y) d y-\gamma \int_{\partial A}\left(\mathbf{m}_{A} \cdot \mathbf{n}_{A}\right)(y) R_{k}^{(\delta)}(x-y) d s_{y} \\
& -\gamma \int_{\partial B}\left(\mathbf{m}_{B} \cdot \mathbf{n}_{B}\right)(y) R_{k}^{(\delta)}(x-y) d s_{y}
\end{aligned}
$$

for all $x \in \mathbb{R}^{d}$. The volume integral in (3.18) can be estimated as follows: Note that $R_{k}^{(\delta)}(x-y)$ is supported on $\mathbb{R}^{d} \backslash B_{\delta / 2}(x)$ and that $\left|R_{k}^{(\delta)}(x-y)\right| \leq c|x-y|^{-(d-1)}$ for some constant $c>0$. Since $\nabla \cdot \mathbf{m}_{A \cup B}$ is essentially bounded on $A \cup B$, the volume integral in (3.18) converges uniformly to the corresponding volume integral of $\mathbf{H}_{A \cup B}(x)$ as $\delta \rightarrow 0$, cf. e.g. [Scha, Proposition 12].

On compact subsets of the smooth portions of $\partial A$, the surface integrals converge uniformly; on the remaining parts of $\partial A$, one has convergence in $L^{1}(\partial A)$, cf. [Scha, Lemma 11]. The limit of $\mathbf{H}_{A \cup B}^{(\delta)}$ is $\overline{\mathbf{H}}_{A \cup B}(x)=\frac{1}{2}\left(\mathbf{H}_{A \cup B}^{+}+\mathbf{H}_{A \cup B}^{-}\right)(x)$, where $\mathbf{H}_{A \cup B}^{+}$and $\mathbf{H}_{A \cup B}^{-}$are the outer and inner trace of $\mathbf{H}_{A \cup B}$ on $\partial A$ with respect to $A$. By the transition condition in (3.2) and since $\mathbf{H}_{A \cup B}=-\nabla u_{A \cup B}$, we have $\left(\mathbf{H}_{A \cup B}^{+}(x)-\mathbf{H}_{A \cup B}^{-}(x)\right) \cdot \mathbf{n}_{A}(x)=-\gamma\left(\mathbf{m}_{B}-\mathbf{m}_{A}\right) \cdot \mathbf{n}_{A}(x)$ for almost every $x \in \partial A$, with $\mathbf{m}_{B}=0$ on $\partial A \backslash(\partial A \cap \partial B)$. Hence, $\overline{\mathbf{H}}_{A \cup B}(x)=\frac{\gamma}{2}\left(\left(\mathbf{m}_{A}-\mathbf{m}_{B}\right) \cdot \mathbf{n}_{A}\right)(x) \mathbf{n}_{A}(x)+\mathbf{H}_{A \cup B}^{-}(x)$ almost everywhere on $\partial A$ and in $L^{1}(\partial A)$ [Scha, Theorem 13].

Since $\nabla \cdot \mathbf{m}_{A} \in L^{\infty}(A)$ and $\mathbf{m}_{A} \cdot \mathbf{n}_{A} \in L^{\infty}(\partial A)$, we can take the limit as $\delta \rightarrow 0$ in (3.17), which implies

$$
\begin{aligned}
\lim _{\delta \rightarrow 0} \lim _{\ell \rightarrow \infty} \mathbf{F}^{\operatorname{long}(\ell, \delta)}= & \int_{A}\left(-\nabla \cdot \mathbf{m}_{A}\right) \mathbf{H}_{A \cup B} d x+\int_{\partial A}\left(\mathbf{m}_{A} \cdot \mathbf{n}_{A}\right) \mathbf{H}_{A \cup B}^{-} d s_{x} \\
& +\frac{\gamma}{2} \int_{\partial A}\left(\mathbf{m}_{A} \cdot \mathbf{n}_{A}\right)\left(\left(\mathbf{m}_{A}-\mathbf{m}_{B}\right) \cdot \mathbf{n}_{A}\right) \mathbf{n}_{A} d s_{x} .
\end{aligned}
$$

Moreover, since $\nabla \mathbf{H}_{A \cup B} \in L^{1}(A)$ by [Scha, Theorem 16], an integration by parts in (3.19) gives (3.14).

Remark 3.3. (a) A comparison of (3.14) and (3.1) shows

$$
\mathbf{F}^{\text {long }}(A, B)=\mathbf{F}^{\mathrm{Br}}(A, B)-\frac{\gamma}{2} \int_{\partial A \cap \partial B}\left(\mathbf{m}_{A} \cdot \mathbf{n}_{A}\right)\left(\mathbf{m}_{B} \cdot \mathbf{n}_{A}\right) \mathbf{n}_{A} d s_{x} .
$$

(b) If, moreover, $\mathbf{m}_{A}$ and $\mathbf{m}_{B}$ are constant magnetization fields, (3.7) yields

$$
\begin{aligned}
\mathbf{F}_{\text {const }}^{\text {long }}(A, B) & =\mathbf{F}_{\text {const }}^{\mathrm{Br}}(A, B)-\frac{\gamma}{2} \int_{\partial A \cap \partial B}\left(\mathbf{m}_{A} \cdot \mathbf{n}_{A}\right)\left(\mathbf{m}_{B} \cdot \mathbf{n}_{A}\right) \mathbf{n}_{A} d s_{x} \\
& =-\gamma \int_{\partial A}\left(\mathbf{m}_{A} \cdot \mathbf{n}_{A}\right)(x) \oint_{\partial B}\left(\mathbf{m}_{B} \cdot \mathbf{n}_{B}\right)(y) \nabla N(x-y) d s_{y} d s_{x} .
\end{aligned}
$$

(c) Note that $\mathbf{F}^{\text {long }}$ does not depend on the specific choice of $\varphi^{(\delta)}$. Given the definition of $\mathbf{F}^{\lim }$, which is independent of $\varphi^{(\delta)}$, it follows that $\mathbf{F}^{\text {short }}$ also has to be independent of $\varphi^{(\delta)}$. 
Next, we consider the short range contribution $\mathbf{F}^{\text {short }}$ to the limit force:

Theorem 3.4. Under Assumption $\mathcal{A}, \mathbf{F}^{\text {short }}(A, B):=\lim _{\delta \rightarrow 0} \lim _{\ell \rightarrow \infty} \mathbf{F}^{\operatorname{short}(\ell, \delta)}(A, B)$ exists, and there holds

$$
\mathbf{F}^{\text {short }}(A, B)=\frac{1}{2} \sum_{i, j, p=1}^{d}\left(S_{i j 1 p}, \ldots, S_{i j d p}\right) \int_{\partial A \cap \partial B}\left(\mathbf{m}_{A}\right)_{i}\left(\mathbf{m}_{B}\right)_{j}\left(\mathbf{n}_{A}\right)_{p} d s_{x}
$$

where $\left(\mathbf{m}_{A}\right)_{i},\left(\mathbf{m}_{B}\right)_{j}$ and $\left(\mathbf{n}_{A}\right)_{p}$ denote the $i$-th, $j$-th, and $p$-th component of the vectors $\mathbf{m}_{A}, \mathbf{m}_{B}$ and $\mathbf{n}_{A}$, respectively, and $\mathbf{m}_{B}$ is the outer trace of $\mathbf{m}_{B}$ on $\partial A$ with respect to $A$. Moreover,

$$
S_{i j k p}:=-\gamma \lim _{\delta \rightarrow 0} \lim _{\ell \rightarrow \infty} \sum_{z \in B_{\delta}(0) \cap \frac{1}{\ell} \mathcal{L} \backslash\{0\}}\left(\partial_{i} \partial_{j} P_{k}^{(\delta)}(z)\right) z_{p} \frac{1}{\ell^{d}} .
$$

Remark 3.4. Note that the sum in (3.22) depends neither on the magnetizations nor on the geometries of the magnets, but only on the underlying lattice $\mathcal{L}$. In particular, $\left(S_{i j k p}\right)_{i, j, k, p=1, \ldots, d}$ is not identically zero in general. See [Sch05] for a discussion of the values of $S_{i j k p}$ for $d=3$ and of the corresponding additional force terms, cf. also [PPS]. For $d=2$, an analogous discussion is given in Appendix A.

Proof of Theorem 3.4. First, note that $\partial_{i} \partial_{j} P_{k}^{(\delta)}(x-y)$ in (3.12) grows like $|x-y|^{-(d+1)}$ and thus is hypersingular. This difficulty can be dealt with as follows: A change of variables via $y=x+z$ and a reorganization of the sum yield

$$
\begin{aligned}
\mathbf{F}_{k}^{\text {short }(\ell, \delta)} & =\gamma \sum_{x \in \bar{A} \cap \frac{1}{\ell} \mathcal{L}} \sum_{y \in B \cap \frac{1}{\ell} \mathcal{L}} \partial_{i} \partial_{j} P_{k}^{(\delta)}(x-y)\left(\mathbf{m}_{A}^{(\ell)}\right)_{i}(x)\left(\mathbf{m}_{B}^{(\ell)}\right)_{j}(y) \\
& =\gamma \sum_{x \in \bar{A} \cap \frac{1}{\ell} \mathcal{L}} \sum_{\substack{z \in B_{\delta}(0) \cap \frac{1}{\ell} \mathcal{L} \backslash\{0\} \\
x+z \in B}} \partial_{i} \partial_{j} P_{k}^{(\delta)}(-z)\left(\mathbf{m}_{A}^{(\ell)}\right)_{i}(x)\left(\mathbf{m}_{B}^{(\ell)}\right)_{j}(x+z) \\
& =-\gamma \sum_{z \in B_{\delta}(0) \cap \frac{1}{\ell} \mathcal{L} \backslash\{0\}} \partial_{i} \partial_{j} P_{k}^{(\delta)}(z) \sum_{x \in A_{z} \cap \frac{1}{\ell} \mathcal{L}}\left(\mathbf{m}_{A}^{(\ell)}\right)_{i}(x)\left(\mathbf{m}_{B}^{(\ell)}\right)_{j}(x+z)
\end{aligned}
$$

with $A_{z}:=\{x \in \bar{A} \mid x+z \in B\}$, where we have again used the summation convention.

Note that the volume of $A_{z}$ is of the order $z$, which suggests that the resulting sum will be singular. Since the unit cell which is associated to a lattice point in $A_{z}$ may not be contained in $A_{z}$, the number of points in $A_{z}$ cannot be naively compared with the volume of $A_{z}$. However, this obstacle can be circumvented by exploiting the lattice structure. To that end, we require the following result [Schb, Proposition 1]:

Proposition 3.5. Let $A$ and $B$ satisfy Assumption $\mathcal{A}$. Fix $0<\delta \ll 1$ and $z \in B_{\delta}(0) \cap \frac{1}{\ell} \mathcal{L} \backslash\{0\}$. Moreover, let $f: A_{z} \rightarrow \mathbb{R}$ be Lipschitz continuous. Then, there exists an $\ell_{0} \in \mathbb{N}$ such that for all $\ell \geq \ell_{0}$

$$
\left|\frac{1}{\ell^{d}} \sum_{x \in A_{z} \cap \frac{1}{\ell} \mathcal{L} \backslash\{0\}} f(x)-\int_{\partial A \cap \partial B} f(x)\left(\mathbf{n}_{A}(x) \cdot z\right)_{+} d s_{x}\right| \leq C|z|^{\frac{4}{3}} .
$$

Here, $(\cdot)_{+}:=\max \{0, \cdot\}$, and the constant $C$ only depends on $\sup (f)$, on the Lipschitz constant of $f$, and on the geometries of $A$ and $B$. 
With $f(x)=\left(\mathbf{m}_{A}\right)_{i}(x)\left(\mathbf{m}_{B}\right)_{j}(x+z)$, it follows from Proposition 3.5 and from the Lipschitz continuity of $\mathbf{m}_{A}$ and $\mathbf{m}_{B}$ that

$$
\mathbf{F}_{k}^{\text {short }(\ell, \delta)}=-\gamma \sum_{z \in B_{\delta}(0) \cap \frac{1}{\ell} \mathcal{L} \backslash\{0\}} \partial_{i} \partial_{j} P_{k}^{(\delta)}(z) \frac{1}{\ell^{d}} \int_{\partial A \cap \partial B}\left(\mathbf{m}_{A}\right)_{i}(x)\left(\mathbf{m}_{B}\right)_{j}(x)\left(\mathbf{n}_{A}(x) \cdot z\right)_{+} d s_{x}
$$

to leading order. The higher-order terms in (3.24) converge to zero if one first takes the limit as $\ell \rightarrow \infty$ and then the limit as $\delta \rightarrow 0$ :

$$
\begin{aligned}
\sum_{z \in B_{\delta}(0) \cap \frac{1}{\ell} \mathcal{L} \backslash\{0\}}\left|\partial_{i} \partial_{j} P_{k}^{(\delta)}(z)\right||z|^{\frac{4}{3}} \frac{1}{\ell^{d}} & \leq c \sum_{z \in B_{\delta}(0) \cap \frac{1}{\ell} \mathcal{L} \backslash\{0\}}|z|^{-(d+1)}|z|^{\frac{4}{3}} \frac{1}{\ell^{d}} \\
& \stackrel{\ell \rightarrow \infty}{\longrightarrow} c \int_{B_{\delta}(0)}|z|^{-d+\frac{1}{3}} d z \leq c \delta^{\frac{1}{3}} .
\end{aligned}
$$

Given the symmetry properties of the kernel $P_{k}^{(\delta)}$ and the fact that $z \in B_{\delta}(0) \cap \frac{1}{\ell} \mathcal{L} \backslash\{0\}$ implies $-z \in B_{\delta}(0) \cap \frac{1}{\ell} \mathcal{L} \backslash\{0\}$ (since $\mathcal{L}$ is a Bravais lattice), we obtain

$$
\sum_{z \in B_{\delta}(0) \cap \frac{1}{\ell} \mathcal{L} \backslash\{0\}} \partial_{i} \partial_{j} P_{k}^{(\delta)}(z)\left(\mathbf{n}_{A}(x) \cdot z\right)_{+}=\frac{1}{2} \sum_{z \in B_{\delta}(0) \cap \frac{1}{\ell} \mathcal{L} \backslash\{0\}} \partial_{i} \partial_{j} P_{k}^{(\delta)}(z)\left(\mathbf{n}_{A}(x) \cdot z\right) .
$$

Thus, substituting (3.25) into (3.24), we find

$$
\mathbf{F}_{k}^{\text {short }(\ell, \delta)}=-\frac{\gamma}{2} \sum_{z \in B_{\delta}(0) \cap \frac{1}{\ell} \mathcal{L} \backslash\{0\}} \partial_{i} \partial_{j} P_{k}^{(\delta)}(z) z_{p} \frac{1}{\ell^{d}} \int_{\partial A \cap \partial B}\left(\mathbf{m}_{A}\right)_{i}(x)\left(\mathbf{m}_{B}\right)_{j}(x)\left(\mathbf{n}_{A}\right)_{p}(x) d s_{x}
$$

up to terms of higher order. The convergence of the sum in (3.22) follows from [Sch05, Lemma 5] for $d=3$ and from [Schb, Theorem 13] for $d=2$, respectively. Hence, we finally obtain (3.21).

Corollary 3.6. Under Assumption $\mathcal{A}, \mathbf{F}^{\lim }(A, B)$ as introduced in (3.13) is well-defined, and there holds

$$
\mathbf{F}^{\lim }(A, B)=\mathbf{F}^{\text {long }}(A, B)+\mathbf{F}^{\text {short }}(A, B)
$$

Remark 3.5. For constant magnetization fields $\mathbf{m}_{A}$ and $\mathbf{m}_{B}$, Equation (3.26) reads

$$
\begin{aligned}
\mathbf{F}_{\text {const }}^{\text {lim }}(A, B) & =\mathbf{F}_{\text {const }}^{\text {long }}(A, B)+\mathbf{F}_{\text {const }}^{\text {short }}(A, B) \\
& =\mathbf{F}_{\text {const }}^{\text {long }}(A, B)+\frac{1}{2} \sum_{i, j, p=1}^{d}\left(S_{i j 1 p}, \ldots, S_{i j d p}\right)\left(\mathbf{m}_{A}\right)_{i}\left(\mathbf{m}_{B}\right)_{j} \int_{\partial A \cap \partial B}\left(\mathbf{n}_{A}\right)_{p} d s_{x} .
\end{aligned}
$$

If, additionally, $A$ and $B$ are nested, i.e., if $\partial A \cap \partial B=\partial A$ holds, $\mathbf{F}_{\text {const }}^{\text {short }}(A, B)$ vanishes, since an integration by parts shows $\int_{\partial A \cap \partial B}\left(\mathbf{n}_{A}\right)_{p} d s_{x}=\int_{A} \partial_{p} 1 d x=0$ in that case.

Next, we summarize a few concluding remarks on the force formulae $\mathbf{F}, \mathbf{F}^{\mathrm{Br}}$, and $\mathbf{F}^{\mathrm{lim}}$, which will be useful for our numerical experiments and the interpretation thereof, see [PPS].

Remark 3.6. (a) A change in the sign of the magnetization of one body changes the sign of the forces. Moreover, observe that the law of "actio equals reactio" holds for all formulae under consideration, cf. [Sch02, Appendix B].

(b) In (2.4), (3.7) and (3.27), the norm of the forces scales like the product of the norms of the magnetizations, since we assume the magnetizations to be constant fields on the two bodies, respectively. 
We end this section with a remark on how Assumption $\mathcal{A}$ on the magnetic domains $A$ and $B$ can be generalized.

Remark 3.7. In the present setting, Assumption $\mathcal{A}$ is primarily required to ensure that the results of [Schb, Scha] can be applied. There, $A$ and $B$ are assumed to be Lipschitz domains with piecewise $\mathcal{C}^{1,1}$ boundaries, cf. [Schb, Definition 2] for a precise definition. Moreover, additional, rather technical conditions are imposed. For the sake of brevity, we only outline those conditions here and refer to [Schb] and [Scha] for details.

(1) $\bar{A} \cup \bar{B}$ satisfies an outer cone property [Schb, Assumption $\mathcal{A}_{1}$ ];

(2) $\partial A, \partial B$, and $\partial A \cup \partial B$ satisfy a so-called non-degeneracy condition $(S)$ that controls the number of isolated points which have the same tangent vector, cf. [Schb, Definition 3];

(3) $\partial A \cap \partial B$ satisfies a so-called neighborhood estimate which allows one to bound volumes of tubes about relative boundaries of portions of $\partial A \cap \partial B$, see [Schb, Definition 4].

In particular, these requirements are satisfied if $A$ and $B$ are polygonal Lipschitz domains with finitely many corners or edges. For further examples of domains satisfying the requirements (1)(3), the reader is referred to [Sch05, p. 236].

If the above weaker assumptions on $A$ and $B$ are imposed instead of Assumption $\mathcal{A}$, we obtain Theorems 3.1, 3.3, and 3.4 as well as the corresponding corollaries in an analogous manner. Finally, we emphasize that the restriction to $d=2$ and $d=3$ is made for physical reasons only, and that our mathematical analysis is valid in any dimension greater or equal than two.

Acknowledgment. The research of NP was supported partly by the Austrian Science Fund FWF under grant P15274 and partly by the U.S. National Science Foundation under grant DMS-0109427. Part of this work was performed while AS was affiliated with the Institute of Analysis, Dynamics, and Modeling at the University of Stuttgart. Moreover, AS was supported by the RTN network MRTN-CT-2004-50522.

\section{Appendix A. Evaluation of $S_{i j k p}$ FOR $d=2$}

In this appendix, we evaluate $S_{i j k p}$ as defined in (3.22) for $d=2$. We prove that $\left(S_{i j k p}\right)_{i, j, k, p=1,2}$ is not identically zero in general; cf. [Sch02] for the analogous result when $d=3$. As mentioned in Section 3 already, $S_{i j k p}$ does not depend on the specific choice of $\varphi^{(\delta)}$. This allows us to reformulate $S_{i j k p}$ so that it can be conveniently evaluated numerically.

Rescaling $z \in \mathcal{L}$ by $\ell$ and taking into account that $-z \in \mathcal{L}$, one can show [Schb, Theorem 13]

$$
S_{i j k p}=-\gamma \lim _{\delta \rightarrow 0} \lim _{\ell \rightarrow \infty} \sum_{z \in B_{\ell \delta}(0) \cap \mathcal{L} \backslash\{0\}}\left(\partial_{i} \partial_{j} P_{k}^{(\ell \delta)}(z)\right) z_{p}=-\gamma \lim _{n \rightarrow \infty} \sum_{z \in B_{n}(0) \cap \mathcal{L} \backslash\{0\}}\left(\partial_{i} \partial_{j} P_{k}^{(n)}(z)\right) z_{p},
$$

where we have used the fact that $\partial_{k} N$ is homogeneous of degree -1 when $d=2$, cf. Remark 3.2.

Now, recall that there holds $P_{k}^{(n)}=\varphi^{(n)} \partial_{k} N$ for any sequence $\left\{\varphi^{(n)}\right\}_{n \in \mathbb{N}}$ of functions which satisfy (3.11). In particular, one can choose a sequence of one-dimensional functions $\phi_{n} \in \mathcal{D}(\mathbb{R})$ with $\left.\phi_{n}\right|_{\{t<n / 2\}}=1$ and $\left.\phi_{n}\right|_{\{t>n\}}=0$ and define $\varphi^{(n)}(z):=\phi_{n}(|z|)$. Then, $\partial_{k} \varphi^{(n)}(z)=\phi_{n}^{\prime}(|z|) \frac{z_{k}}{|z|}$, and a somewhat lengthy but elementary calculation gives

$$
\begin{aligned}
-\partial_{i} \partial_{j} P_{k}^{(n)}(z)= & \underbrace{\frac{\gamma}{2 \pi}\left(-\frac{2}{|z|^{4}} \phi_{n}(|z|)+\frac{1}{|z|^{3}} \phi_{n}^{\prime}(|z|)\right)}_{=: f(|z|)}\left(\delta_{i j} z_{k}+\delta_{i k} z_{j}+\delta_{j k} z_{i}\right) \\
& +\underbrace{\frac{\gamma}{2 \pi}\left(\frac{8}{|z|^{2}} \phi_{n}(|z|)-\frac{5}{|z|} \phi_{n}^{\prime}(|z|)+\phi_{n}^{\prime \prime}(|z|)\right)}_{=: g(|z|)} \frac{z_{i} z_{j} z_{k}}{|z|^{4}} .
\end{aligned}
$$


Moreover, by the definition of $\phi_{n}$, there holds

$$
f(|z|)=\left\{\begin{array}{ll}
\frac{\gamma}{2 \pi} \frac{2}{|z|^{4}} & \text { if }|z| \leq \frac{n}{2}, \\
0 & \text { if }|z| \geq n
\end{array} \quad \text { and } \quad g(|z|)= \begin{cases}-\frac{\gamma}{2 \pi} \frac{8}{|z|^{2}} & \text { if }|z| \leq \frac{n}{2} \\
0 & \text { if }|z| \geq n\end{cases}\right.
$$

By (A.2), $\partial_{i} \partial_{j} P_{k}^{(n)}(z)$ is symmetric in the indices $i, j$, and $k$. Hence, only four types of terms in (A.1) need to be considered,

$$
S_{i i i k}^{(n)} \quad \text { and } \quad S_{i k k k}^{(n)}
$$

as well as

$$
S_{i i k k}^{(n)} \text { and } S_{k k k k}^{(n)},
$$

where $i \neq k$. The sums in (A.3) are antisymmetric in $z_{i}$ and zero if $z_{i}=0$. Therefore, the two terms in (A.3) turn out to be zero for all $n \in \mathbb{N}$ if $\mathcal{L}$ is the square lattice $\mathbb{Z}^{2}$. Consequently, the limit in (A.1) is then also zero. As for (A.4), the terms that occur in the sums in (A.1) are given by

$$
f(|z|) z_{k}^{2}+g(|z|) \frac{z_{i}^{2} z_{k}^{2}}{|z|^{4}} \quad \text { and } \quad 3 f(|z|) z_{k}^{2}+g(|z|) \frac{z_{k}^{4}}{|z|^{4}}
$$

respectively, and are symmetric in all components of $z$. Our aim is to show that these terms cannot be zero simultaneously, at least if $\mathcal{L}=\mathbb{Z}^{2}$. To that end, we split the sum over all lattice vectors in the ball $B_{n}=B_{n}(0)$ into a sum over all lattice vectors in $B_{n} \backslash B_{n / 2}$ and a sum over $B_{n / 2}=B_{n / 2}(0)$. A change of variables yields

$$
\sum_{z \in B_{n} \backslash \bar{B}_{n / 2} \cap \mathcal{L}}\left(\partial_{i} \partial_{j}\left(\varphi^{(n)} \partial_{k} N\right)(z)\right) z_{p}=\sum_{z \in B_{1} \backslash \bar{B}_{1 / 2} \cap \frac{1}{n} \mathcal{L}}\left(\partial_{i} \partial_{j}\left(\varphi^{(1)} \partial_{k} N\right)(z)\right) z_{p} \frac{1}{n^{2}},
$$

where we have again made use of the fact that $\partial_{k} N$ is homogeneous of degree -1 when $d=2$. The right hand side of (A.5) is a Riemann sum and converges to $\int_{B_{1} \backslash B_{1 / 2}}\left(\partial_{i} \partial_{j}\left(\varphi^{(1)} \partial_{k} N\right)(z)\right) z_{p} d z$ as $n \rightarrow \infty$. Let $\nu$ denote the outer normal to $\partial\left(B_{1} \backslash B_{1 / 2}\right)$. Then, given the definition of $\varphi^{(1)}$, an integration by parts shows that

$$
\begin{aligned}
\int_{B_{1} \backslash B_{1 / 2}}\left(\partial_{i} \partial_{j}\left(\varphi^{(1)} \partial_{k} N\right)(z)\right) z_{p} d z \\
=\int_{\partial\left(B_{1} \backslash B_{1 / 2}\right)}\left\{\nu_{i}(z)\left(\partial_{j}\left(\varphi^{(1)} \partial_{k} N\right)(z)\right) z_{p}-\nu_{j}(z)\left(\varphi^{(1)} \partial_{k} N\right)(z) \delta_{i p}\right\} d s_{z} \\
=-\int_{\partial B_{1 / 2}}\left\{\frac{z_{i}}{|z|}\left(\partial_{j} \partial_{k} N(z)\right) z_{p}-\frac{z_{j}}{|z|} \partial_{k} N(z) \delta_{i p}\right\} d s_{z} \\
=\frac{\gamma}{2 \pi} \int_{\partial B_{1 / 2}}\left\{\frac{z_{i} z_{p}}{|z|^{3}}\left(\delta_{j k}-2 \frac{z_{j} z_{k}}{|z|^{2}}\right)-\frac{z_{j} z_{k}}{|z|^{3}} \delta_{i p}\right\} d s_{z} .
\end{aligned}
$$

This implies

$$
\int_{B_{1} \backslash B_{1 / 2}}\left(\partial_{k} \partial_{k}\left(\varphi^{(1)} \partial_{k} N\right)(z)\right) z_{k} d z=-\frac{\gamma}{2 \pi} \int_{\partial B_{1 / 2}} 2 \frac{z_{k}^{4}}{|z|^{5}} d s_{z}=-\frac{3 \gamma}{4} \quad \text { for } i=j=k=p
$$

and

$$
\int_{B_{1} \backslash B_{1 / 2}}\left(\partial_{i} \partial_{i}\left(\varphi^{(1)} \partial_{k} N\right)(z)\right) z_{k} d z=-\frac{\gamma}{2 \pi} \int_{\partial B_{1 / 2}} 2 \frac{z_{i}^{2} z_{k}^{2}}{|z|^{5}} d s_{z}=-\frac{\gamma}{4} \quad \text { for } i=j \neq k=p .
$$


It remains to evaluate

$$
s_{\iota \iota k k}^{(n)}:=-\sum_{z \in \bar{B}_{n / 2} \cap \mathcal{L} \backslash\{0\}}\left(\partial_{\iota} \partial_{\iota} \partial_{k} N(z)\right) z_{k}
$$

for $\iota=k$ and $\iota \neq k$, respectively. Since $N$ is the fundamental solution of Laplace's equation, there holds $\sum_{\iota=1}^{2} s_{\iota \iota k k}^{(n)}=0$. Hence, $s_{i i k k}^{(n)}=-s_{k k k k}^{(n)}$ for every $n \in \mathbb{N}$. By Theorem 3.4 and the preceding analysis, we know that $s_{i i k k}^{(n)}$ and $s_{k k k k}^{(n)}$ converge in $\mathbb{R}$ as $n \rightarrow \infty$. We define

$$
\mathcal{S}:=\lim _{n \rightarrow \infty} s_{k k k k}^{(n)}=-\frac{\gamma}{2 \pi} \lim _{n \rightarrow \infty} \sum_{z \in \bar{B}_{n / 2} \cap \mathcal{L} \backslash\{0\}} \frac{2 z_{k}^{2}}{|z|^{4}}\left(3-4 \frac{z_{k}^{2}}{|z|^{2}}\right),
$$

which gives $\lim _{n \rightarrow \infty} s_{i i k k}^{(n)}=-\mathcal{S}$. Finally,

$$
S_{k k k k}=\mathcal{S}+\frac{3 \gamma}{4} \quad \text { and } \quad S_{i i k k}=-\mathcal{S}+\frac{\gamma}{4}
$$

Consequently, $S_{k k k k}$ and $S_{i i k k}$ cannot be simultaneously zero, and it follows that the tensor $S=$ $\left(S_{i j k p}\right)_{i, j, k, p=1,2}$ is not identically zero. In particular, if $\mathcal{L}=\mathbb{Z}^{2}$, a brute-force numerical computation shows $\mathcal{S} \approx \frac{\gamma}{2 \pi} 2.50765$.

\section{REFERENCES}

[Bob00] S. Bobbio. Electrodynamics of Materials: Forces, Stresses, and Energies in Solids and Fluids. Academic Press, San Diego, 2000.

[Bro66] W.F. Brown. Magnetoelastic Interactions. Springer-Verlag, Berlin, 1966.

[Dör68] W. Döring. Das elektromagnetische Feld, volume 2 of Einführung in die theoretische Physik. Walter de Gruyter\&Co., Berlin, 1968.

[DPG96] A. DeSimone and P. Podio-Guidugli. On the Continuum Theory of Deformable Ferromagnetic Solids. Arch. Ration. Mech. Anal., 136:201-233, 1996.

[EM90] A.C. Eringen and G.A. Maugin. Electrodynamics of continua. Springer-Verlag, New York, 1990.

[ES98] Yu.V. Egorov and M.A. Shubin. Foundations of the Classical Theory of Partial Differential Equations. Springer-Verlag, Berlin, 1998.

[LL84] L.D. Landau and E.M. Lifschitz. Electrodynamics of continuous media, volume 8 of Course of theoretical physics. Pergamon Press, Oxford, second edition, 1984. Second Russian edition revised by Lifshitz and L.P. Pitaevskiı̌.

[MS02] S. Müller and A. Schlömerkemper. Discrete-to-continuum limit of magnetic forces. C.R. Acad. Sci. Paris, Ser. I, 335:393-398, 2002.

[PPS] N. Popović, D. Praetorius, and A. Schlömerkemper. Analysis and Numerical Simulation of Magnetic Forces between Rigid Polygonal Bodies. Part II: Numerical Simulation. Max Planck Institute for Mathematics in the Sciences, Leipzig, Preprint 64/2006, to appear in Contin. Mech. Thermodyn.

[PPS05] N. Popović, D. Praetorius, and A. Schlömerkemper. Magnetic Force Formulae for Magnets at Small Distances. PAMM Proc. Appl. Math. Mech., 5:631-632, 2005.

[Scha] A. Schlömerkemper. About solutions of Poisson's equation with transition condition in non-smooth domains. Max Planck Institute for Mathematics in the Sciences, Leipzig, Preprint 53/2006, to appear in Z. Anal. Anwend.

[Schb] A. Schlömerkemper. Lattice approximation of a surface integral and convergence of a singular lattice sum. Max Planck Institute for Mathematics in the Sciences, Leipzig, Preprint 86/2005, to appear in Asymptot. Anal.

[Sch02] A. Schlömerkemper. Magnetic forces in discrete and continuous systems. PhD thesis, University of Leipzig, Leipzig, 2002.

[Sch05] A. Schlömerkemper. Mathematical derivation of the continuum limit of the magnetic force between two parts of a rigid crystalline material. Arch. Rational Mech. Anal., 176:227-269, 2005. 
Boston University, Department of Mathematics and Statistics and Center for BioDynamics, 111 Cummington Street, Boston, MA 02215, U.S.A.

Vienna University of Technology, Institute for Analysis and Scientific Computing, Wiedner HauptStrasse 8-10, A-1040 Vienna, Austria

Corresponding author. Max Planck Institute for Mathematics in the Sciences, Inselstrasse 22-26, D-04103 Leipzig, Germany, Email: schloem@mis.mpg.de, Tel: +49-341-9959-547, Fax: +49-341-9959-633 